\title{
Contextual Deletion of Object and Ambiguity in Machine Translation
}

\author{
Gloria Álvarez Benito \\ Universidad de Sevilla \\ J. Gabriel Amores Carredano \\ Universidad de Sevilla
}

\begin{abstract}
Constructions surfacing as intransitive are highly ambiguous in English. They may be the reflection of a true intransitive verb or, rather, the result of one of the linguistic processes which derive an intransitive construction from a canonical transitive one (e.g. contextual deletion of an understood object, deletion of an optional reflexive marker or disergativization). This type of lexical/structural ambiguity poses many problems in Machine Translation. In this article we offer a possible solution which has been implemented in JULIETTA, a Lexical-Functional Grammar-based machine translation prototype.
\end{abstract}

\section{Introduction}

It is common practice in English to omit the object complement from the sentence because of economy of language or simply to avoid redundancy. The latent object can be recovered through the context, either situational or linguistic:

(1) Shake well before use!

(2) Are you going to play tennis or just watch?

In sentence (1) the content of the covert object is recaptured through the situational context. The deleted object does not appear in the sentence structure and it is the situation that helps us understand the message. In sentence (2), however, the linguistic context provides the necessary information to interpret the sentence correctly; the deleted object of the verb watch does not appear at surface structure level in order to avoid redundancy.

Contextual deletion is closely related to the problem of ambiguity. The reason is that most verbs do not have one lexical entry only. If we delete the object in a monotransitive 
pattern, we get a pseudo-intransitive structure as a result. ${ }^{1}$ Now, if the verb which appears in that sentence has another lexical entry in addition to the monotransitive structure in which the verb is intransitive, then we have two possible interpretations. There are many cases in which we are not sure of the right reading. We do not know for sure whether the verb is intransitive by nature or whether it is an originally monotransitive one which has undergone object deletion.

\section{Structural Ambiguity}

As a general rule, ambiguity is usual with those verbs having at least two different lexical entries. In one of them the verb is monotransitive and in the other it appears as intransitive due to one of the following reasons: (a) inherently reflexive verbs; (b) inherently reciprocal verbs; (c) ergative verbs.

\subsection{Reflexive verbs}

In English we may distinguish between inherently and non-inherently reflexive verbs. Within the former we find those verbs which do not necessarily require a reflexive marker or proform in order to imply reflexivity (myself, yourself, himself, herself, etc.). In the latter group we can pigeon-hole all those verbs in which a reflexive proform is obligatory to show reflexivity:

(3) He shaved very early

(4) He cut himself with a knife

The verb in (3) is inherently reflexive and that is why it may appear in an intransitive structure. However, the verb in (4) is non-inherently reflexive and therefore we have a monotransitive structure, since the reflexive pronoun functions as the object required by the verb.

The group of verbs which may present ambiguity is the one made up of inherently reflexive verbs. Verbs within this group may have a double reading. On the one hand we can interpret the structure in which they appear as intransitive by nature and, on the other hand, we may assume that the structure is not intransitive but pseudo-intransitive after the deletion of the object at surface structure level. Consider the following sentences:

(5) I stood in the sitting room while he shaved

(6) I always wash twice a day

In the above-mentioned examples, there are two possible interpretations:

(5a) I stood in the sitting room while he shaved himself $_{i}$

(5b) I stood in the sitting room while he shaved someone $_{j}$

(6a) $I_{i}$ always wash myself twice a day

(6b) $I_{i}$ always wash something $g_{k}$ twice a day 
As we can see, in the two sentences under (a) we interpret the verbs as inherently reflexive, whereas in the two sentences under (b) we understand that there has been a contextual deletion of an object and consequently the verbs are monotransitive by nature.

\subsection{Reciprocal verbs}

The process of reciprocity is very similar to reflexivity. As in reflexivity, we have to distinguish between inherently and non-inherently reciprocal verbs. The difference between them is that the latter require the reciprocal proform each other or one another (when more than two participants are implied), whereas the former do not require any proform or marker to imply reciprocity:

(7) They met

(8) They saw each other

In these examples, the non-inherently reciprocal verb (8) appears in a monotransitive structure, the reciprocal proform functioning as the direct object. However, the inherently reciprocal verb (7) does not require any proform and therefore it appears in an intransitive structure. ${ }^{2}$ As we mentioned in the case of reflexive verbs, it is inherently reciprocal verbs that may appear in ambiguous structures, as the following example illustrates:

(9) John and Claire are always fighting

The double reading of this sentence lies in the fact that we can interpret the verb fight either as inherently reciprocal or as a non-reciprocal verb. In the first interpretation there is an association of subjects but we can split the association with the following result:

(9a) John is always fighting with Claire

and we can have the reversible structure:

(9a') Claire is always fighting with John

In its second reading we do not interpret John and Claire as an association of subjects but as a plural subject, so it is a monotransitive structure where there has been object deletion. The interpretation for this sentence would be as follows:

(9b) John and Claire are always fighting with someone else

\subsection{Ergative verbs}

The last group of intransitive constructions comprises those verbs commonly known as ergative. ${ }^{3}$ In these verbs, the ergative element in a transitive structure has been deleted and the original object takes the syntactic function of subject keeping its semantic role. Consider the following sentence: 
(10) Andrew broke the bottle

The syntactic pattern of this sentence is SVO. If we delete the ergative element "Andrew," the affected constituent surfaces as the subject, as illustrated in (11):

(11) The bottle broke

For obvious reasons, there are many cases which do not present any problem of interpretation, for example:

(12) The door opened

(13) The bell rang

(14) The river narrows after 10 metres

(15) The shops have closed

The problem arises in sentences in which, as well as with inherently reflexive verbs and inherently reciprocal verbs, we may have a double reading. On the one hand we may interpret that there has been disergativization, which causes the structure to be intransitive and on the other hand, we may also assume that there is a latent object at surface structure level and so the structure is monotransitive. Let us now analyze examples (16) and (17):

(16) Mary was cooking

(17) Mary moved

Again, these two sentences are ambiguous because of the reasons above-mentioned. The different interpretations would be:

(16a) Mary was cooking (something)

(16b) Mary was being cooked

(17a) Mary moved (herself)

(17b) Mary was moved (by something)

Obviously, the ambiguity of these sentences is restricted to the syntactic level. If we had semantic information provided by the context, such sentences could easily be disambiguated. That is, the constituents of the sentence we consider ambiguous would be assigned different semantic roles depending on the interpretation, while at surface level they share the same syntactic configuration. In the above-mentioned examples, Mary would be agent in the first reading (17a), whereas in the second reading (17b) Mary would be affected by the action identified by the verb.

To sum up, we are dealing with three linguistic processes (contextual deletion of an understood object, deletion of an optional reflexive marker in object position, and disergativization) that derive a surface intransitive construction from a transitive one. The problem of ambiguity arises when we cannot identify what type of process took place, i.e. contextual deletion of an object or deletion of an optional reflexive marker. In these cases, the appropriate context is necessary to resolve the ambiguity. In the next sections we will see how this type of problem may be resolved within the context of Machine Translation. 


\section{Machine Translation and Ambiguity}

Ambiguity is one of the bottle-necks of Machine Translation (MT), and of any computer program that tries to understand human language. Many linguistic phenomena result in some kind of ambiguity: words, for example, may belong to more than one syntactic category (syntactic homonymy), and the program must be able to decide which is the correct one. Categorial ambiguity is very productive in English, as the different uses of the word round show in the following examples ${ }^{4}$ :

(18) Liverpool were eliminated in the first round

(19) The cowboy started to round up the cattle

(20) I want to buy a round table

(21) We are going on a cruise round the world

(22) A bucket of cold water soon brought him round

(23) The tree measured six feet round

Ambiguity can also be structural if different phrasal configurations result in different interpretations. So, for example, in I saw a girl with a telescope we may mean that I used a telescope to see the girl, or that the girl I saw had a telescope. The kind of problems under consideration in the present article exhibit a mixture of lexical and structural ambiguity. First, we are dealing with verbs that may be used in several subcategorization frames (for example there would be at least two entries for break in (11), depending on the semantic role of the subject). Second, we are trying to analyze an intransitive construction which is highly ambiguous in the sense that we ignore a priori whether it corresponds to a true intransitive verb or to an intransitive verb entry derived from a transitive one through one of the linguistic processes mentioned above.

In an MT application we find some additional problems. First, MT systems make little use of extrasentential context to resolve cases of ambiguity due to the complexity involved in implementing this type of strategy. Rather, most systems work on a sentence-bysentence basis, making some sort of best-guess according to different heuristics. By and large, it will be the responsibility of the post-editor to check that the system made the right decision. Otherwise, he/she will report the problem to the system developer for its subsequent adjustment.

Another problem comes from the purely linguistic nature of the processes we are studying here. As we have pointed out, these constructions are derived through the application of lexical rules which generate one entry from another. This mechanism poses some interesting challenges to any MT system. On the one hand, the system must be able to capture linguistic generalizations without having to resort to unnecessary coding or repetitions in the dictionary. On the other hand, the type of linguistic information to be manipulated by the system has to be rich enough to allow for subtle distinctions stemming from the presence of thematic roles, semantic features in the arguments involved, grammatical functions, subcategorization frames, etc. The system must be capable of assigning different representations to superficially similar constructions making use of this information concurrently. 


\section{Overview of JULIETTA, an LFG-Based MT Prototype}

Any MT developer would agree that it is necessary to incorporate sophisticated linguistic information into the system if we want to capture linguistic generalizations of the sort outlined here. The problem comes in finding the right amount of linguistics without affecting the robustness and performance of the system. Within the past three years we have been developing JULIETTA (Amores Carredano), an English to Spanish MT prototype based on the postulates of Lexical-Functional Grammar (LFG) (Bresnan, ed.). LFG belongs to the family of unification-based grammars (Shieber), currently one of the most widely accepted approaches in the field of Computational Linguistics.

LFG was developed in the late 1970s by Joan Bresnan and Ron Kaplan. It emerged with the aim of serving as the grammatical basis for a computationally precise and psychologically realistic model of human language (Sells 135, Bresnan, ed. Introduction and 173ff). LFG assigns two syntactic representations to each sentence, each level conveying a different type of information and obeying different constraints.

The constituent structure (c-structure) is a phrase structure tree representing surface constituency configurations, generated by the classical context-free rewriting rules. Roughly speaking, it follows the principles of X-Bar Theory (Jackendoff). This structure serves as the input to the phonological component of the grammar. ${ }^{5}$ The second level of representation is the functional structure $(f$-structure): this represents the surface grammatical relations that are expressed in the sentence as well as grammatical features. It is the input to semantic interpretation.

The greatest advantage of using LFG in the context of MT lies precisely in this double representation assigned to every sentence. Intuitively, the f-structure serves ideally as the input for transfer in a conventional transfer-based MT system. ${ }^{6}$ While the c-structure conveys language-dependent information which is discarded after analysis, grammatical relations provide language-independent information of the sort needed during transfer. Instead of manipulating trees that still reflect the source language ordering, LFG manipulates feature-value pairs, which are more abstract objects and where order is not relevant. The f-structure generated by an LFG grammar may serve as the input to the transfer component, and the f-structure resulting from transfer may generate the corresponding target c-structure. Graphically, we may illustrate the overall architecture of the system as in figure 1 (see Appendix 1). To give an idea of the kind of result that will be obtained following these principles, we reproduce the actual output of JULIETTA for a simple test sentence in figure 2 (see Appendix 1). ${ }^{7}$

\section{Strategy of Analysis and Lexical Rules}

The strategy we have followed combines the use of lexical and grammar rules. Lexical rules are the main function-changing mechanism in LFG. Consider the dative-shift alternation of verbs like give in English.

(24) The boy gave a cake to the girl 
(25) The boy gave the girl a cake

In LFG, each of these examples shows a different lexical form, namely

$$
\begin{gathered}
\left(24^{\prime}\right) \text { give }<(\mathrm{SUBJ}),(\mathrm{OBJ}),\left(\mathrm{OBL}_{\mathrm{GO}}\right)> \\
\left(25^{\prime}\right) \text { give }<(\mathrm{SUBJ}),(\mathrm{OBJ}),(\mathrm{OBJ})> \\
\text { give }<\mathrm{AG}, \mathrm{TH}, \mathrm{GO}>
\end{gathered}
$$

The different grammatical functions (GFs) in the lexical forms are not ordered according to their surface linear order, but according to the underlying predicate argument structure (PAS) (Bresnan ed. 150). Instead of declaring that the verb give has these two (among other) lexical forms, LFG stipulates that there exists a lexical rule that derives (25') from $(24 ')$. This rule is repeated below.

\subsection{Dative Shift}

$$
\begin{aligned}
& \left(\mathrm{OBL}_{\mathrm{GO}}\right) \rightarrow(\mathrm{OBJ}) \\
& (\mathrm{OBJ}) \rightarrow(\mathrm{OBJ} 2)
\end{aligned}
$$

Lexical rules account for those phenomena that are explained by transformations or movement in other generative frameworks. Some other examples of lexical rules are the following:

\subsection{Intransitivization}

$$
(\mathrm{OBJ}) \rightarrow \phi
$$

This would relate (26) and (27) below

(26) The boy ate a cake

(27) The boy ate at $3.00 \mathrm{pm}$

In both examples eat is logically a two-place predicate eat $<1,2>$, with two different lexical forms

$$
\begin{aligned}
& \left(26^{\prime}\right) \text { eat }<(\text { SUBJ }),(\text { OBJ })> \\
& \left(27^{\prime}\right) \text { eat }<(\text { SUBJ }), \phi>
\end{aligned}
$$

(27') shows clearly that the second argument has no surface syntactic realization. Note that $\phi$ is not a GF, it simply shows that this argument slot corresponds to no GF in surface structure. ${ }^{9}$ 


\subsection{Passivization}

Passivization is another lexical rule in LFG, and perhaps the strongest claim in the theory. ${ }^{10}$

$$
\begin{aligned}
& (\mathrm{SUBJ}) \rightarrow\left(\mathrm{OBL}_{\mathrm{AG}}\right) / \phi \\
& \mathrm{Ag} \\
& (\mathrm{OBJ}) \rightarrow(\text { SUBJ }) \\
& \text { Th } \\
& (\uparrow \mathrm{PART})=\text { pass }
\end{aligned}
$$

This rule states that the OBJ function is replaced by SUBJ, and SUBJ by either $\mathrm{OBL}_{\mathrm{AG}}$ or $\phi$ (thus accounting for both long and short passives), with the semantic constraint that the subject has to be an agent and the object a theme. Unlike other lexical rules, passive has a morphological consequence in that its output is a passive participle.
(28) The boy ate a cake
(29) The cake was eaten by the boy
eat<(SUBJ),(OBJ)>
eat $<\left(\mathrm{OBL}_{\mathrm{AG}}\right),(\mathrm{SUBJ})>$
(30) The cake was eaten
eat $<\phi$,(SUBJ)>

As regards its implementation, these rules are not included in the dictionary but in the parser. Thus, the system starts trying with the original verb entry. If it does not apply, the appropriate lexical rule generates another entry in case the phrasal configuration predicts that we may be facing a linguistic generalization defined as a lexical rule.

\section{Strategy of Analysis}

The general strategy works as follows. When the parser encounters an intransitive construction, it must choose among the following possibilities in this order.
(a) Pure intransitive verbs (e.g. such as work or arrive)
(b) Ergative verbs (e.g. he broke the bottle $\rightarrow$ the bottle broke)
(c) Reciprocal verbs (e.g. someone 1 met someone $2 \rightarrow$ they met)
(d) Reflexive verbs (e.g. he never shaves before lunch)
(e) Contextual deletion (e.g. he usually eats at two)

Let us go through each of them to see how the parser discriminates among the different possibilities.

(a) The first option is to try a purely intransitive verb. This will be so if the dictionary returns a frame of the type $\langle$ (SUBJ) $>$. Verbs such as run, which can be used as transitive or intransitive (he runs a book shop/he runs very fast), do not pose any problem, since the transitive option would have been tried earlier by a different rule. 
(b) The next possibility is to check whether the verb is ergative (the bottle broke). The parser will generate a new entry if the following conditions are met: the verb is transitive, its subject is defined as an agent and its object as a theme (break, for example). ${ }^{11}$ These conditions are checked on the original entry of the verb in the dictionary. The next step is to look at the features of the noun phrase realized as subject (the bottle in the example above). This subject must not be animate. ${ }^{12}$ If it is the case, the parser changes the subcategorization frame of the verb from $\langle$ (SUBJ), (OBJ) $\rangle$ to $\langle$ (SUBJ), $\phi\rangle$, replaces the thematic role of the subject from agent to theme, and adds the feature erg:yes. ${ }^{13}$ The resulting f-structure for the example above would be as shown in figure 3 (see Appendix 1). ${ }^{14}$

(c) Reciprocity. An example would be the boy and the girl met. Again, the basic condition is that the verb be originally transitive, but this time the thematic roles must be the same for the subject and the object. We understand that in inherently reciprocal verbs, if someone A meets someone B, none of them is less agentive or less thematic. Therefore, we assume that they share the same thematic role. The discussion is open as to whether what is shared is agentivity or thematicity. We have assumed that they share thematicity since LFG adopts a localist approach to thematic roles. In localist theories, if there is only one role to be assigned, it must be theme (Gruber 38). If these conditions are met, the subcategorization frame will be $\langle$ (SUBJ), $\phi\rangle$, and the feature ref 1 : yes will be added. An additional condition is that the subject must be plural (e.g. they) or through the coordination of singular subjects (e.g. the boy and the girl).

d) Reflexivity. The example would be he never shaves before lunch. As we have already mentioned, the parser cannot have access to extrasentential information in order to disambiguate between contextual deletion of an object and deletion of a reflexive marker. Since most native speakers opt for a reflexive reading, ${ }^{15}$ we have implemented this option as the most plausible. Here again, the reflexive feature refl : yes is necessary to generate Spanish correctly.

e) Contextual deletion (he usually eats at two). If none of the previous cases apply, the parser assumes that it is facing a process of contextual deletion. A simple change in the subcategorization frame from $<(\mathrm{SUBJ}),(\mathrm{OBJ})>$ to $<(\mathrm{SUBJ}), \phi>$ suffices to indicate that the object is not superficially realized.

Sentences with reciprocal and reflexive verbs where the reflexive marker is present pose no problem from an analysis point of view. The anaphoric element is analyzed as an object and its refl:yes feature is inherited by the verb. Information about the object in English is eliminated during transfer so that it is not generated in Spanish. The diagrams in figure 4 (see Appendix 1) show the analysis and transfer representations for the sentence they saw each other.

Note also that nothing has been said about binding conditions on anaphora, and how it is resolved in LFG. We are working here with simple sentences, assuming that the 
subject is always the antecedent of the anaphora. Obviously, this is an oversimplification of such a complex problem. For a proposal within the framework of LFG, see Dalrymple.

\section{Results}

This section presents the results of implementing this strategy in JULIETTA. We display a typical session in Prolog that shows the input test sentences and their corresponding translations generated by the system.

| ?- try.
$>>$ The boy ate the cake.
El niño se comió el pastel.

yes.

| ?- try.

$\gg$ The boy ate.

El niño comió.

yes.

| ? - try.

>> The boy broke the bottle.

El niño rompió la botella.

yes.

1?- try.

$\gg$ The bottle broke.

La botella se rompió.

yes.

1?- try.

$\gg$ The boy and the girl met.

El niño y la niña se encontraron.

yes.

| ?- try.

$>$ They met each other.

se encontraron.

yes.

| ?- try.

> The boy met the girl.

El niño encontró a la niña.

yes.

| ?- try.

$>$ The boy and the girl saw each other.

El niño y la niña se vieron.

yes. 
|?- try.

> The boys saw one another.

Los niños se vieron.

yes.

1? - try.

$\gg$ The boy shaved himself.

El niño se afeitó.

|?- try.

$\gg$ The boy cut himself with a knife.

El niño se cortó con un cuchillo.

yes.

|? - try.

$>$ The boy never shaves before lunch.

El niño nunca se afeita antes del almuerzo.

yes.

\section{Conclusion}

In this article we have looked at five intransitive constructions in English. Four of them are the result of some linguistic process that derived an intransitive construction from a canonical transitive one. We have seen that this type of structural ambiguity poses many problems for MT systems. In section 4 we described JULIETTA, a prototype system based on LFG. Section 6 explained how a combination of grammar rule ordering and the generation of new verb entries through lexical rules provided a valid solution to help the parser discriminate among the different options. Finally, section 7 showed the results of implementing this strategy in JULIETTA. These results lead to the conclusion that LFG is not only a suitable theory for the analysis component of an MT system, but also that its levels of linguistic representation fit perfectly into the architecture of a transfer-based MT system.

\section{Notes}

1. With the term pseudo-intransitive we refer to those verbs which are transitive by nature but which appear without any object at surface structure level. In that sense, they present the same structure as an intransitive verb. Such is the case of sentences like she is reading, where the verb read appears without its object because it is not relevant and consequently the verb presents the same structure as an intransitive one.

2. Obviously, it is worthwhile indicating that not all inherently reciprocal verbs require the proform. In fact, there are inherent reciprocal verbs which require the proform in order to be grammatical, as we can see in the sentence Spain and France border each other. There are some other verbs in which the proform appears as a redundant element (e.g. they met each other). We should also point out that some non-inherent reciprocal verbs may appear without any proform, as for example $I$ saw them kissing. 
3. Ergative verbs are the ones which allow the speaker to describe the action either from the point of view of the element which performs the action or from the point of view of the element affected by the action. They are also called unaccusative verbs, especially in Relational Grammar.

4. Examples taken from Hutchins and Somers (85). in $\mathrm{LFG}$.

5. The c-structure is the only phrase structure representation. There is no 'deep' c-structure

6. For an overview of basic approaches in Machine Translation, see Hutchins and Somers (ch. 4).

7. The change in font is meant to indicate that we are reproducing computer output.

8. The version of lexical rules we are describing here corresponds to what may be considered as "early' LFG. See Bresnan ("Monotonicity") and the references cited therein for a new approach currently under development in LFG.

9. We can easily compare this rule with Deletable Roles, Covert Roles and the Derivational System in Case Grammar (Cook 197, 184, 202). Also, certain semantic restrictions apply to lexical rules in order to block overgeneration.

10. Ch. 1 of Bresnan ed., "The Passive in Lexical Theory" details the motivations for a lexicalist approach to passivization, as opposed to a transformationalist theory.

11. We are aware of the limitations of this strategy. For example, nothing would prevent the system to analyze a sentence such as *the stone kicked, suppossedly derived from the transitive the boy kicked the stone. A more refined semantic classification of verbs is needed than the one outlined here.

12. JULIETTA is equipped with a semantic network which is consulted during analysis and transfer. Every noun included in the dictionary must also be included in the semantic network.

13. The feature erg:yes will trigger reflexivity during transfer to Spanish, but something different if we are translating into another language. This is so in order to keep the f-structure as language-independent as possible.

14. We would also like to point out here that other computer-assisted translation systems fail to translate this type of sentence correctly (Âlvarez Benito and Torreblanca López).

15. This may be so because shave belongs to the semantic class of bodily-care verbs.

\section{Works Cited}

Amores Carredano, J. G. de. A Lexical-Functional Grammar-Based Machine Translation System for Medical Abstracts. Ph. D. Thesis. Universidad de Sevilla, 1992.

Álvarez Benito, G., and Torreblanca López, M. M. "Los errores sintácticos en los sistemas de traducción automática: análisis del programa Translate." Actas del I Congreso de Lengua y Literatura Anglo-Norteamericana. Ciudad Real, forthcoming.

Bresnan, J., ed. The Mental Representation of Grammatical Relations. Cambridge: MIT P, 1982.

Bresnan, J. "Monotonicity and the Theory of Relation Changes in LFG." Proceedings of the Seoul International Conference on Natural Language Processing. Seoul, 1990.

Cook, W. Case Granmar Theory. Washington: Georgetown University Press, 1989.

Dalrymple, M. "An LFG Account of Anaphoric Binding Constraints." Manuscript. Xerox-PARC and CSLI. Stanford University, 1991.

Gruber, J. S. Lexical Structures in Syntax and Semantics. Amsterdam: North Holland, 1976.

Hutchins, W., and H. Somers. An Introduction to Machine Translation. London: Academic Press, 1992. 
Jackendoff, R. S. X-Syntax: A Study of Phrase Structure. Linguistic Inquiry Monograph 2. Cambridge: MIT Press, 1977.

Sells, P. Lectures on Contemporary Syntactic Theories. CSLI Lecture Notes 3. Stanford, 1985.

Shicber, S. M. An Introduction to Unification-Based Approaches to Grammar. Stanford: CSLI, 1986.

\section{Appendix 1}

Source c-structure

$S-\rightarrow \underset{\uparrow S u b j=\downarrow}{N P} \quad$ VP

$\left[\begin{array}{l}\text { PRED }: \mathrm{X} \\ \text { TENSE }: \mathrm{Y} \\ \text { SUBJ }[\mathrm{Z}]\end{array}\right]$

Source f-structure
Target c-structure

$S^{\prime} \rightarrow N_{\uparrow S u b j=\downarrow}^{N P^{\prime}} \quad \begin{array}{ll}T P^{\prime} \\ \uparrow=\downarrow\end{array}$

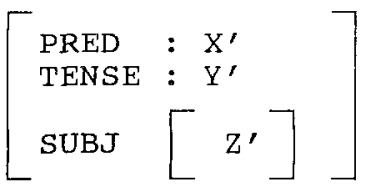

Target f-structure

ANALYSIS ------> , TRANSFER -----> GENERATION

Figure 1

LFG and MT

\section{English Input}

The boy ate a cake.

English c-structure

$\mathrm{s}(\operatorname{clh}(\mathrm{cl}(\mathrm{np}(\operatorname{detp}(\operatorname{det} 2(\operatorname{det}(\operatorname{the})))$, n2 (n1 (n (boy)))),

$\operatorname{vph}(\operatorname{vp}(v g(v(a t e))$,

$n p(\operatorname{detp}(\operatorname{det} 2(\operatorname{det}(a)))$,

n2 (n1 (n(cake)))))))))

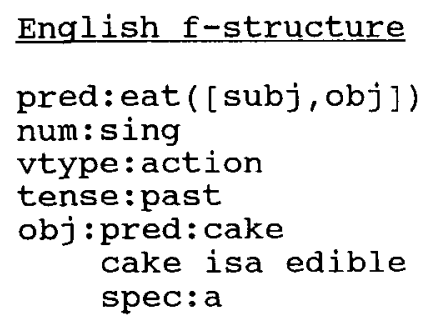

English f-structure

pred: eat ([subj, obj])

num:sing

vtype:action

tense:past

obj : pred: cake

cake isa edible

spec:a

Spanish f-structure

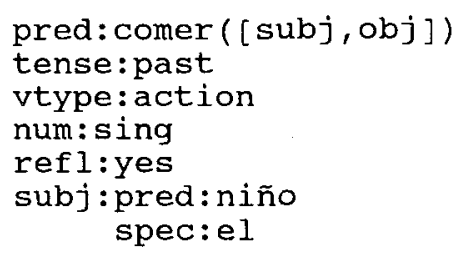




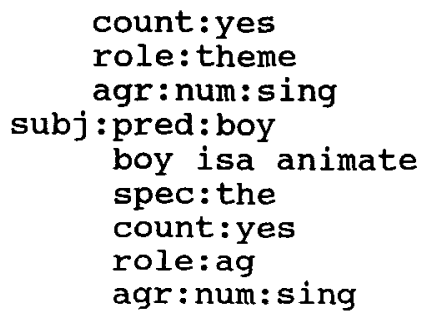

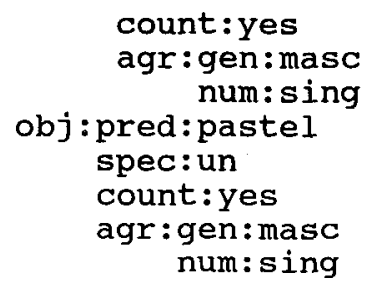

\section{Spanish c-structure}

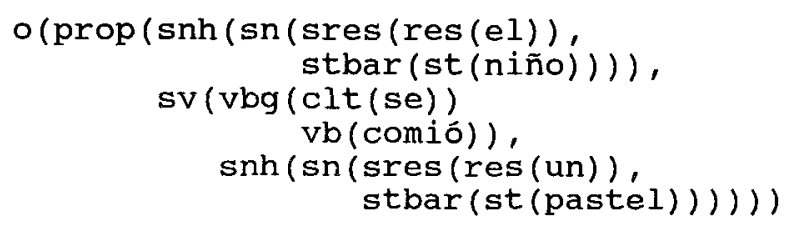

Spanish output

El niño se comió un pastel.

Figure 2

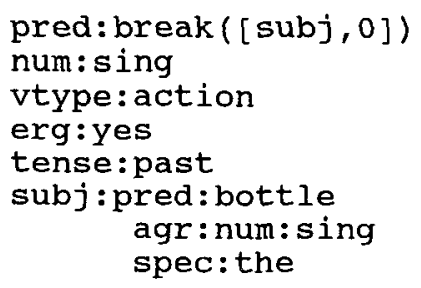

Figure 3

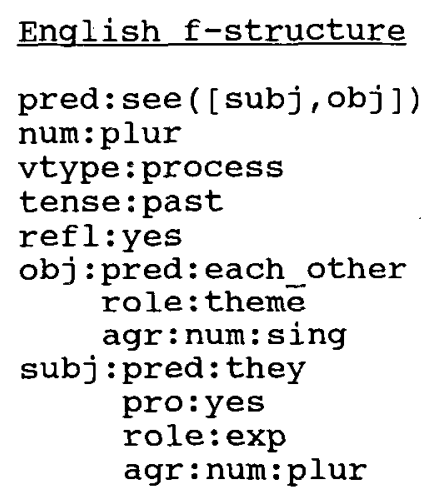

